\title{
A novel DC-DC converter and LDO cascaded circuit with improved dynamic response and loop stability
}

\author{
Hao Zhou ${ }^{1, \text { a) }}$, Hao Min ${ }^{1}$, and Ping Luo ${ }^{2}$
}

Abstract Presented is a new type of DC-DC converter and low dropout voltage regulator (LDO) cascaded circuit, which has improved dynamic response and loop stability. In this novel cascaded circuit, the gate voltage of the pass element of the LDO is quantized and compared with a target value to form an error value to finally control the feedback coefficient of the DC-DC converter. Meanwhile, the adjusting speed of this feedback coefficient can be changed dynamically through the gain compensator, which is minimized to obtain the best loop stability when approaching the steady-state and increased to achieve a fast transient response during large dynamic change. The testing results of the prototype designed to verify the feasibility show that the novel cascaded circuit can increase the response speed by 22 times and optimize the voltage ripple by 1.9 times.

Keywords: DC-DC converter, low dropout voltage regulator (LDO), dynamic response, loop stability

Classification: Electron devices, circuits and modules (silicon, compound semiconductor, organic and novel materials)

\section{Introduction}

With the rapid growth of the demand for various portable devices and wearable electronic products, the demand for high-performance power management chips is also increasing. The efficiency of DC-DC converters can reach more than $90 \%[1,2,3,4,5,6,7,8,9]$. However, its large output ripple limits its applications in sensitive electronic load where additional LDO is required to achieve low power noise and ripple $[10,11,12,13,14,15,16,17,18,19]$. Hybrid methods of switched-mode, switched-capacitor-mode DC-DC, low dropout voltage regulator (LDO) are proposed in recent years [20, 21, 22, 23, 24]. The traditional architecture of the analog switched-mode DC-DC converter with embedded LDO is shown in Fig. 1, the output voltage of the DC-DC converter must meet the dropout voltage requirement of the LDO under maximum load conditions, resulting in low efficiency under general load or light load [25, 26]. In the paper [27, 28, 29], several hybrid DC-DC and LDO cascading methods with digital controlled DC-DC converter shown as Fig. 2 are proposed to improve the performance. In [29], by using the pass element's gate voltage of the LDO as the voltage detection point of the DC-DC converter loop, the

${ }^{1}$ State Key Lab of ASIC \& Systems, Fudan University, Shanghai 201203, People's Republic of China

${ }^{2}$ University of Electronic Science and Technology of China, Chengdu 610054, Chengdu

a) biganalogintegrated@gmail.com

DOI: 10.1587/elex.18.20210072

Received February 10, 2021

Accepted March 10, 2021

Publicized March 22, 2021

Copyedited April 25, 2021

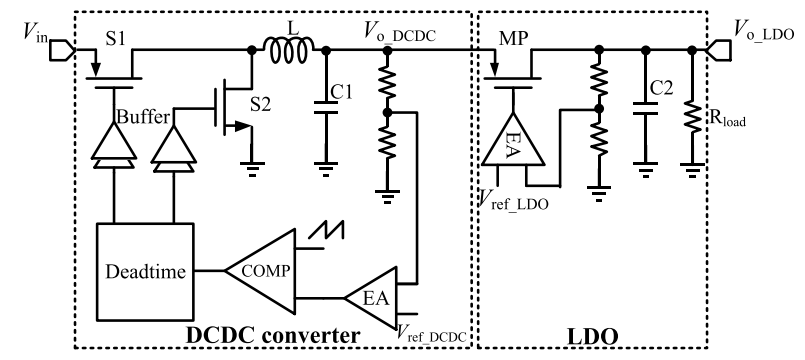

Fig. 1 The traditional architecture of the analog DC-DC converter with embedded LDO.

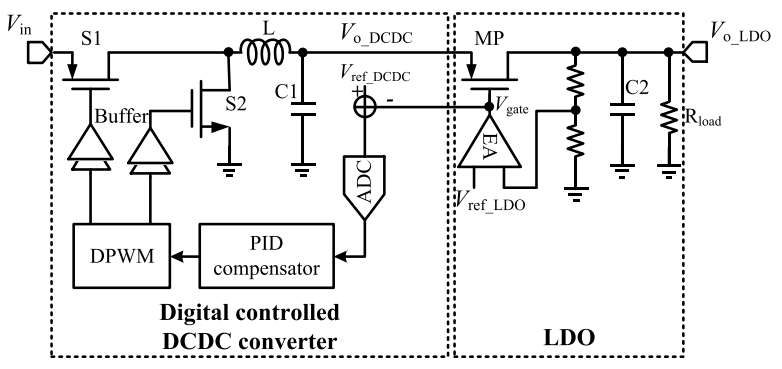

Fig. 2 The traditional architecture of the digital DC-DC converter with embedded LDO.

DC-DC converter can provide just enough dropout voltage for different loads. However, since the loop characteristics of the LDO are added to the feedback loop of the DC-DC converter, the two-loop characteristics become more complex which requires complicated loop compensation. Other papers such as $[28,30]$ also proposed the methods of adaptive change of the dropout voltage. Although the zero/pole of the LDO is avoided to be brought into the DC-DC converter loop, these dropout voltage adjustment methods are not the most efficient for the gate voltage of the LDO's pass element is not detected directly.

In this paper, in order to improve dynamic response and loop stability further, a new type of DC-DC converter and LDO cascaded circuit with the gain compensator is proposed to change the speed of the feedback coefficient of the digital controlled DC-DC converter dynamically.

\section{Working principle of the proposed circuit}

The novel architecture of a digital controlled DC-DC converter and an LDO is illustrated in Fig. 3. A low-power ADC2 is used to quantize the gate voltage $\left(V_{\text {pgate }}\right)$ of the PMOS pass element MP of the LDO. The output of the $\mathrm{ADC} 2$ compares with a target digital value to form the error 


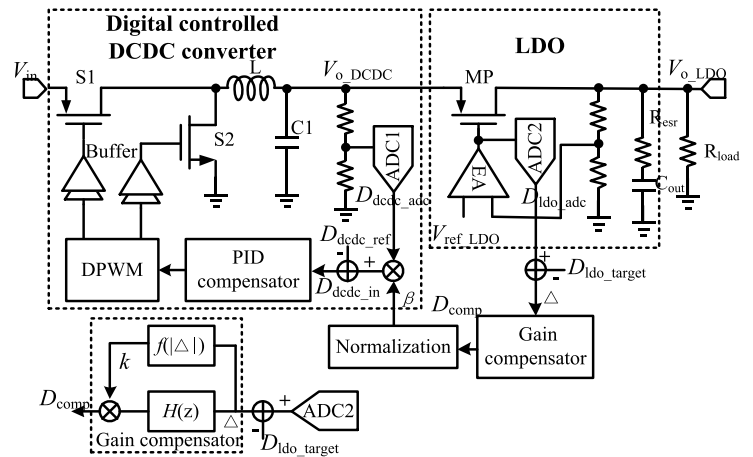

Fig. 3 The novel architecture of the DC-DC converter and LDO cascaded circuit.

value that will be sent to a variable gain compensator (the input voltage of the ADC2 corresponding to this target digital value is the voltage target of the PMOS's gate voltage). The output value of the gain compensator is normalized to form a feedback coefficient that finally controls the feedback of the DC-DC output voltage.

As shown in Fig. 3, the input value of the DC-DC converter's control module can be written as:

$$
D_{\text {dedc_in }}=\beta D_{\text {dedc } \_ \text {adc }}
$$

where $D_{\text {dcdc } \text { in }}$ is the output value of $\operatorname{ADC} 1$ and $\beta$ is the feedback coefficient that can be expressed as:

$$
\beta=\left(2^{N}-D_{\text {comp }}\right) / 2^{N}
$$

where $N$ is the bit number of the compensator output $D_{\text {comp }}$, which can be expressed as:

$$
D_{\text {comp }}=k\left(D_{\text {ldo_adc }}-D_{\text {ldo_target }}\right) H(z)
$$

where $D_{\text {ldo_adc }}$ is the output of ADC2, $D_{\text {ldo_target }}$ is the target digital value of the PMOS's gate voltage and $H(\mathrm{z})$ is the loop filter transfer function, and the loop gain parameter $k$ can be expressed as:

$$
k=f(|\Delta|)=f\left(\left|D_{\text {ldo_adc }}-D_{\text {ldo_target }}\right|\right)
$$

where $f(|\Delta|)$ is a compensation function related to $\mid D_{\text {ldo_adc }}-$ $D_{\text {ldo_target }} \mid$.

According to (1), the value of $\beta$ reflects the corresponding response speed during load switching. According to (2) (4), when $\left|D_{\text {ldo_adc }}-D_{\text {ldo_target }}\right|$ becomes large $(>0.2)$ suddenly (load switching), $k$ needs to be a large number correspondingly to accelerate the dynamic response of the DC-DC converter.

When $\left|D_{\text {ldo_adc }}-D_{\text {ldo_target }}\right|$ is close to zero $(\leq 0.2), k$ needs to be a small number dramatically such that the two loops behave as two approximately independent loops. This conclusion can be obtained through the following proof process.

The complete small-signal model of the proposed circuit is illustrated in Fig. 4. The loop gain of the LDO can be expressed as:

$$
G_{L D O}=g_{m} Z_{o} H A(s)
$$

where $Z_{o}$ is the output impedance of the LDO which can be expressed as:

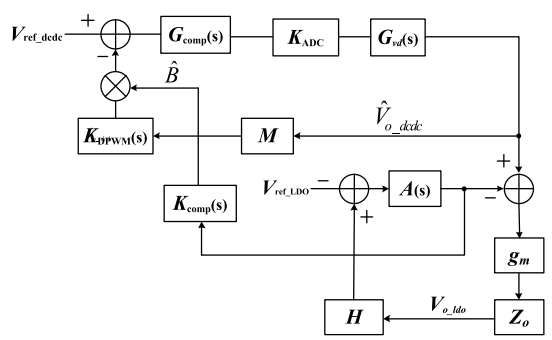

Fig. 4 The small signal mode of the proposed circuit.

$$
Z o=R_{\text {out }} \frac{1+C_{\text {out }} R_{\text {esr }} s}{1+C_{\text {out }}\left(R_{\text {out }}+R_{\text {esr }}\right) s}=R_{\text {out }} \frac{1+\frac{s}{\omega_{E S R}}}{1+\frac{s}{\omega_{\text {out }}}}
$$

And $A(s)$ is the gain of the error amplifier which can be expressed as:

$$
A(s)=\frac{G_{o p}}{1+\frac{s}{\omega_{o p}}}
$$

The gain of the gain compensator can be expressed as

$$
K_{\text {comp }}(s)=\frac{K}{s}
$$

The small-signal of $\beta$ can be expressed as:

$$
\hat{B}=\hat{V}_{O_{-} d c d c} \frac{K_{\text {comp }}(s) G_{L D O}}{1+G_{L D O}}
$$

Then substituting (5) (8) into (9), we can get:

$$
\hat{B} \approx \hat{V}_{o_{-} d c d c} \frac{K}{s} \bullet \frac{1}{1+\frac{s}{A_{\text {LDO } \omega_{\text {out }}}}}
$$

In the main DC-DC loop, the input value $\left(D_{\text {dcdc_in) }}\right.$ before the PID compensator is equal to the sum of the steady-state value and the change value which can be expressed as:

$$
\begin{aligned}
& \hat{V}_{O \_d c d c} M K_{A D C}\left(B_{\text {steady }}+\hat{B}\right) \\
& =\hat{V}_{o \_d c d c} M K_{A D C}\left(B_{\text {steady }}+\hat{V}_{o \_d c d c} \frac{K}{S} \bullet \frac{1}{1+\frac{s}{A_{L D O \omega_{\text {out }}}}}\right)
\end{aligned}
$$

According to (11), when $\mathrm{K}$ is very small, the second term (second-order term) can be ignored at small-signal mode. Then the input value before the PID compensator can be expressed as:

$$
\hat{V}_{O_{-} d c d c} M K_{A D C} B_{\text {steady }}
$$

At this time, the characteristic of the DC-DC loop has nothing to do with LDO. In other words, when $\mathrm{K}$ is very small, the two loops (DC-DC loop and LDO loop) behave as two approximately independent loops. Thus, this topology can maintain the two loops' AC stability when approaching steady-state, and has a quick transient response for a dynamic change.

In order to realize the special properties of $k$, the compensation function $f(|\Delta|)$ can be expressed as:

$$
k=f(|\Delta|)=\frac{4.5}{1+\mathrm{e}^{-1000 \times(|\Delta|-0.2)}}+0.5
$$

And the waveform of the compensation function $f(|\Delta|)$ is illustrated in Fig. 5 showing that the value of $k$ can be switched from 0.5 to 5 according to $\left|D_{\text {ldo_adc }}-D_{\text {ldo_target }}\right|$. 


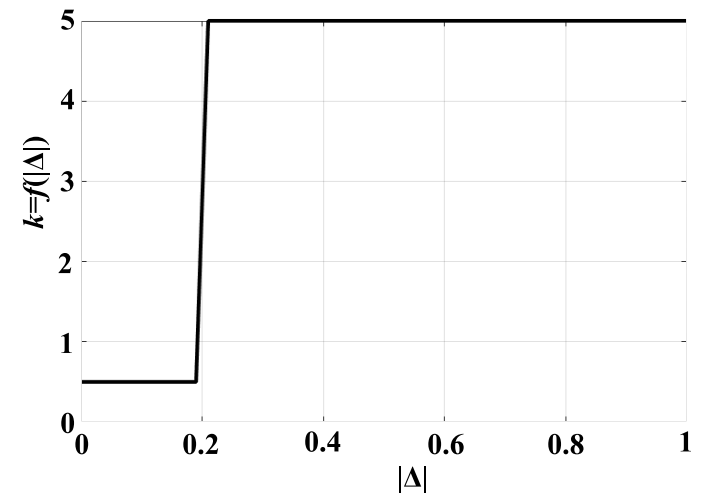

Fig. 5 The compensation function $f(\Delta)$.

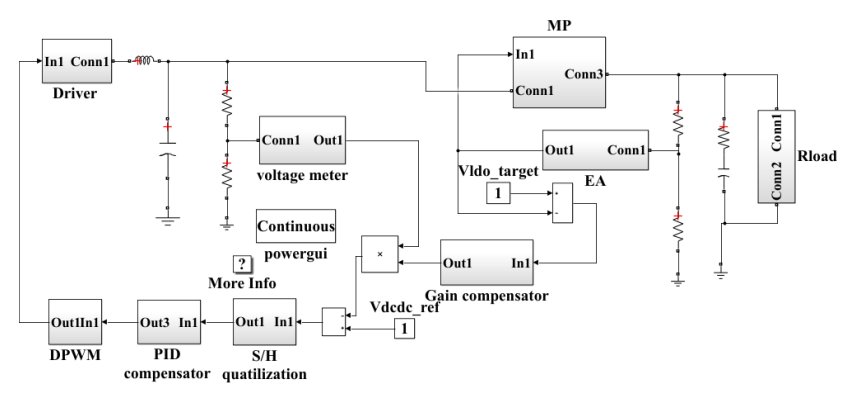

Fig. 6 The Simulink block of the proposed circuit.

\section{Simulation results}

The Simulink block of the proposed cascaded circuit is shown in Fig. 6. And three conditions are simulated to verify the improvement of the proposed cascaded circuit as shown in Fig. 7.

When $\mathrm{k}$ is a fixed value and a small number $(=0.5)$, the simulation result is shown as Fig. 7(a). At this time, the response speed of the circuit is slow $(\Delta t=0.25 \mathrm{~ms})$ when the load is switched from $200 \mathrm{~mA}$ to $300 \mathrm{~mA}$, because the corresponding feedback coefficient $\beta$ is very small.

When $k$ is a fixed value and a large number $(=5)$, the simulation result is shown as Fig. 7(b). The response speed of the circuit becomes fast $(\Delta t=0.03 \mathrm{~ms})$ when the load is switched from $225 \mathrm{~mA}$ to $300 \mathrm{~mA}$, because the corresponding feedback coefficient $\beta$ becomes larger. However, it can be seen from the previous analysis that the characteristic of the DC-DC loop will be affected by LDO when $\mathrm{k}$ is large. Thus, the loop stability of DC-DC is poor at this time.

The simulation result is shown in Fig. 7(c) when the value of $\mathrm{k}$ is determined by the gain compensation. It is shown that the circuit can maintain good stability and fast response speed at the same time.

\section{Experimental results}

A prototype is also designed to verify the feasibility of the system as shown in Fig. 8. The test system employs NCP302025 as the integrated driving circuit of the DC-DC converter, FDV304P as the pass element, and LT1719 as the EA of the LDO. And two ADCs (ADC12020) are employed to provide 8-bit quantization for the gate voltage of

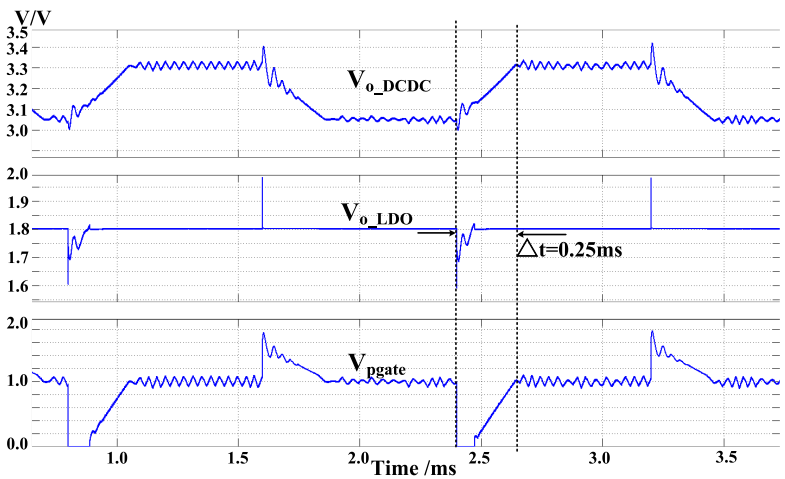

(a)

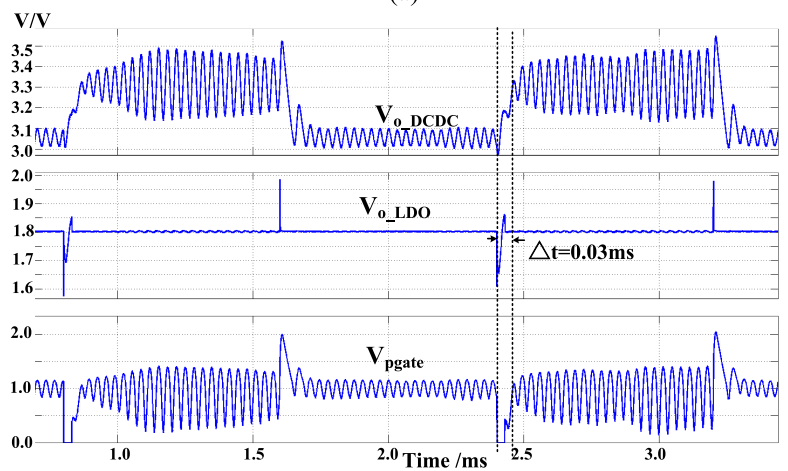

(b)

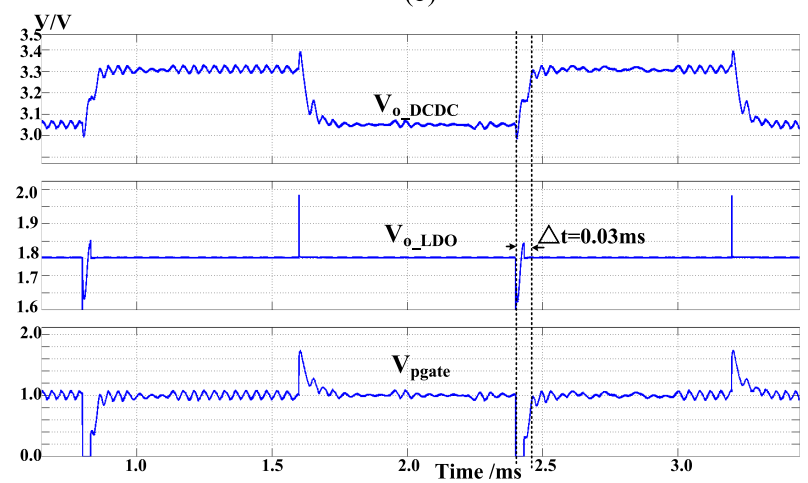

(c)

Fig. 7 The simulation waveforms of the Simulink block. (a) Fixed $k=$ 0.5 ; (b) fixed $k=5$; (c) $k$ with gain compensation.

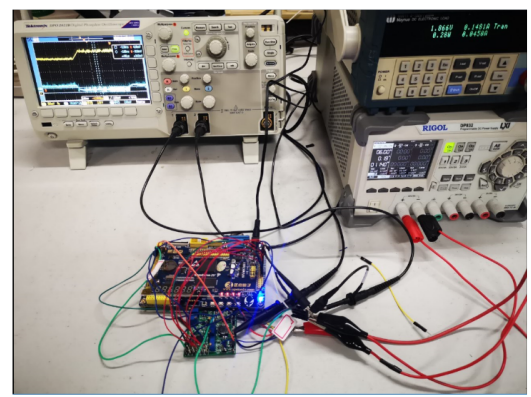

Fig. 8 Test setup of the proposed circuit.

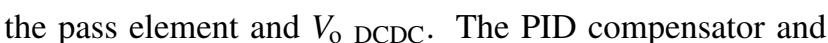
DPWM are realized through FPGA. A load current step between $225 \mathrm{~mA}$ and $300 \mathrm{~mA}$ is applied to the prototype with $6 \mathrm{~V}$ input voltage.

Fig. 9(a) shows the case for fixed $k=0.5$ where the ripple voltage of the LDO is $17 \mathrm{mV} @ 300 \mathrm{~mA}$ and $13 \mathrm{mV} @ 225 \mathrm{~mA}$, while the output voltage of the DC-DC converter varies from 


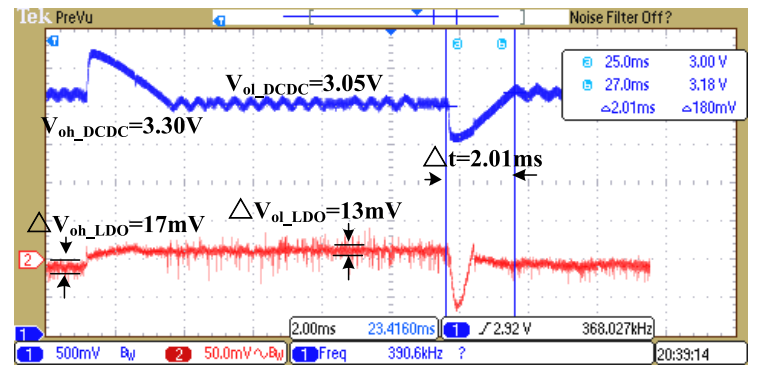

(a)

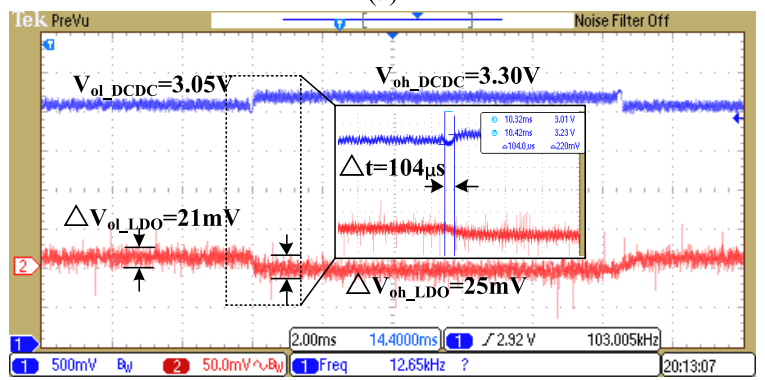

(b)

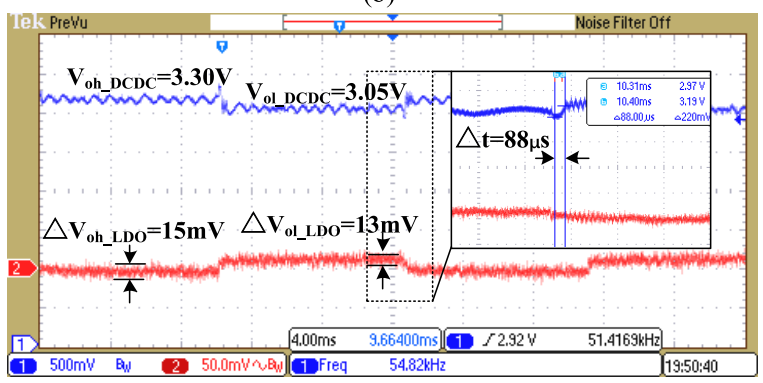

(c)

Fig. 9 The experimental results. (a) Fixed $k=0.5$; (b) fixed $k=5$; (c) with gain compensation.

$3.30 \mathrm{~V}$ to $3.05 \mathrm{~V}$. And when the load is switched, the response time is $2.01 \mathrm{~ms}$.

Fig. 9(b) shows the case for fixed $k=5$ where the ripple voltage of the LDO is 25mV@300mA and 21mV@225mA, and when the load is switched, the response time is only $104 \mu s$.

Fig. 9(c) shows the case for $k$ determined by the gain compensation. The response time of the DC-DC converter is only $88 \mu$ s which is much smaller than the case for $\mathrm{k}=0.5$. While the ripple voltage of the LDO is $15 \mathrm{mV} @ 300 \mathrm{~mA}$ and $13 \mathrm{mV} @ 225 \mathrm{~mA}$, the circuit with gain compensation optimizes the voltage ripple by 1.9 times.

\section{Conclusion}

A feedback coefficient obtained from quantizing and compensating the pass element's gate voltage of the LDO can be used to control the DC-DC converter to provide the adaptive dropout voltage for the cascaded LDO. Furthermore, by adaptively changing the gain of the compensator linking the two loops, good loop stability and fast transient response are both achieved in this system when approaching steady-state and undergoing large dynamic change, respectively.

\section{References}

[1] T. An, et al:: "High-efficiency low-noise pulse-width modulation
DC-DC buck converter based on multi-partition switching for mobile system-on-a-chip applications," IET Power Electronics (2016) 559 (DOI: 10.1049/iet-pel.2014.0731).

[2] S. Shin, et al.: "A 95.2\% efficiency dual-path DC-DC step-up converter with continuous output current delivery and low voltage ripple," IEEE International Solid-State Circuits Conference (2018) 430 (DOI: 10.1109/ISSCC.2018.8310368).

[3] C. Hardy and H. Le: "8.3 A 10.9W 93.4\%-efficient (27W 97\%-efficient) flying-inductor hybrid DC-DC converter suitable for 1-cell (2-cell) battery charging applications," IEEE International Solid State Circuits Conference (2019) 150 (DOI: 10.1109/ ISSCC.2019.8662432).

[4] P. Lan, et al.: "An asynchronous digitally-controlled switching converter with adaptive resolution and dynamic power saving to achieve higher than $93.5 \%$ efficiency between $5 \mathrm{~mA}$ and $250 \mathrm{~mA}$ load," IEEE Asian Solid State Circuits Conference (2011) 45 (DOI: 10.1109/ASSCC.2011.6123584).

[5] S. Kim, et al.: "A 10MHz 2mA-800mA 0.5V-1.5V 90\% peak efficiency time-based buck converter with seamless transition between PWM/PFM modes," IEEE Custom Integrated Circuits Conference (2017) 1 (DOI: 10.1109/CICC.2017.7993619).

[6] Y. Shin, et al.: "Efficiency improvement of dual mode DC-DC buck converter under light load using PTWS with a zero current detector," International Conference on Circuits System and Simulation (2017) 72 (DOI: 10.1109/CIRSYSSIM.2017.8023185).

[7] Y. Ahn, et al:: “A 93.5\% efficiency, 400-mA current-mode dc-dc buck converter with watchdog functions," International SoC Design Conference (2009) 428 (DOI: 10.1109/SOCDC.2009.5423862).

[8] S.J. Kim, et al.: "A $10 \mathrm{MHz} 2 \mathrm{~mA}-800 \mathrm{~mA} 0.5 \mathrm{~V}-1.5 \mathrm{~V} 90 \%$ peak efficiency time-based buck converter with seamless transition between PWM/PFM modes," IEEE Custom Integrated Circuits Conference (2017) 1 (DOI: 10.1109/CICC.2017.7993619).

[9] Y.H. Shin, et al.: "Efficiency improvement of dual mode DC-DC buck converter under light load using PTWS with a zero current detector," International Conference on Circuits System and Simulation (2017) 72 (DOI: 10.1109/CIRSYSSIM.2017.8023185).

[10] Fares, et al.: "Simplified review of DCDC switching noise and spectrum contents," Ph.D. Research in Microelectronics and Electronics (2014) 1 (DOI: 10.1109/PRIME.2014.6872677).

[11] S.T. Kim, et al., "Enabling wide autonomous DVFS in a $22 \mathrm{~nm}$ graphics execution core using a digitally controlled fully integrated voltage regulator,' IEEE J. Solid-State Circuits 51 (2016) 18 (DOI: 10.1109/JSSC.2015.2457920).

[12] M.M. Elkhatib: "A capacitor-less LDO with improved transient response using neuromorphic spiking technique," International Conference on Microelectronics (2016) 133 (DOI: 10.1109/ICM. 2016.7847927).

[13] J. Moon, et al.: "Design of low-power, fast-transient-response, capacitor-less low-dropout regulator for mobile applications," IEICE Electron. Express 13 (2016) 1 (DOI: 10.1587/elex.15.20180651).

[14] Y. Li, et al.: "A 0.45-to-1.2-V fully digital low-dropout voltage regulator with fast-transient controller for near/subthreshold circuits," IEEE Trans. Power Electron. 31 (2016) 6341 (DOI: 10.1109/ TPEL.2015.2506605).

[15] N. Liu, et al.: "A transient-enhanced fully-integrated LDO regulator for SoC application," IEEE International Symposium on Circuits and Systems (2018) 1 (DOI: 10.1109/ISCAS.2018.8351236).

[16] S. Liu, et al.: "Design of self-referenced wide input voltage range LDO using enhanced current mirror buffer and improved lead compensation," IEICE Electron. Express 17 (2020) 1 (DOI: 10.1587/ elex.17.20200120).

[17] X. Qu, et al.: "A fast-transient on-chip LDO with advanced dynamic biasing circuit," IEICE Electron. Express 11 (2014) 1 (DOI: 10.1587/elex.11.20140690).

[18] J. Shi, et al.: "A self-clocked binary-seaching digital low-dropout regulator with fast transient response," IEICE Electron. Express 17 (2020) 1 (DOI: 10.1587/elex.17.20200297).

[19] M. Huang, et al.: "11.5 A 2-phase soft-charging hybrid boost converter with doubled-switching pulse width and shared bootstrap capacitor achieving $93.5 \%$ efficiency at a conversion ratio of 4.5," IEEE International Solid- State Circuits Conference (2020) 198 (DOI: 
10.1109/ISSCC19947.2020.9062902).

[20] R. Magod, et al.: "A 1.24 $\mu$ A quiescent current NMOS low dropout regulator with integrated low-power oscillator-driven charge-pump and switched-capacitor pole tracking compensation," IEEE J. SolidState Circuits 53 (2018) 2356 (DOI: 10.1109/JSSC.2018.2820708).

[21] Y. Lu, et al.: "An NMOS-LDO regulated switched-capacitor DCDC converter with fast-response adaptive-phase digital control," IEEE Trans. Power Electron. 31 (2016) 1294 (DOI: 10.1109/TPEL. 2015.2420572).

[22] W.C. Chen, et al.: "A switchable digital-analog low-dropout regulator for analog dynamic voltage scaling technique," IEEE J. Solid-State Circuits 49 (2014) 740 (DOI: 10.1109/JSSC.2013.2297395).

[23] Z. Xiao, et al.: "A switched-capacitor DC-DC converter with embedded fast NMOS-LDOs achieving low noise, low output voltage ripple and fast response," International Symposium on Integrated Circuits (2014) 1 (DOI: 10.1109/ISICIR.2016.7829698).

[24] F.U. Ahmed, et al.: "A brief overview of on-chip voltage regulation in high-Performance and high-density integrated circuits," IEEE Access. 9 (2021) 813 (DOI: 10.1109/ACCESS.2020.3047347).

[25] M.H. Kamel, et al.: "A hybrid NMOS/PMOS low-dropout regulator with fast transient response for SoC applications," International Conference on Microelectronics (2017) 1 (DOI: 10.1109/ICM. 2017.8268850).

[26] S. Dietrich, et al.: "A $1 \mathrm{mV}$ voltage ripple $0.97 \mathrm{~mm}^{2}$, fully integrated low-power hybrid buck converter," European Solid-State Circuits Conference (2013) 395 (DOI: 10.1109/ESSCIRC.2013.6649156).

[27] K.K. Avalur, et al.: "Automotive hybrid voltage regulator design with adaptive LDO dropout using load-sense technique," Asia Pacific Conference on Circuits and Systems (2015) 240 (DOI: 10.1109/ APCCAS.2014.7032846).

[28] K.K. Avalur, et al.: "A 6-18 V hybrid power management IC with adaptive dropout for improved system efficiency up to $150^{\circ} \mathrm{C}$," IEEE J. Emerg. Sel. Topics Power Electron. 6 (2018) 477 (DOI: 10.1109/JESTPE.2017.2760345).

[29] Z.H. Shen, et al.: "Combination method of DC-DC converter and LDO to improve efficiency and load regulation." Electronics Letters 47 (2011) 615 (DOI: 10.1049/el.2010.7135).

[30] W. Yuan, et al.: "An LDO regulated DC-DC converter with voltage ripple suppression and adaptive dropout voltage control," IEICE Electron. Express 15 (2016) 1 (DOI: 10.1587/elex.15.20180651). 